\title{
Comparação do desempenho dos músculos flexores e extensores de joelho em jovens de esportes coletivos com e sem salto
}

\author{
Knee flexor and extensor muscles performance comparison in youth team sports \\ with and without jumping
}

Comparación del desempeño de los músculos flexores y extensores de la rodilla en jóvenes en deportes colectivos con y sin salto

Vinícius Jardim Oliano', Lilian Pinto Teixeira², Juliana Corrêa Soares ${ }^{3}$, Michele Forgiarini Saccol ${ }^{4}$, Simone Lara $^{5}$

\begin{abstract}
Resumo I O objetivo deste estudo é comparar o desempenho dos músculos flexores e extensores de joelho em jovens atletas de esportes coletivos que utilizam ou não o movimento do salto como gesto característico. Foram incluídos jovens atletas masculinos alocados em dois grupos: grupo salto ( $n=46$, atletas de voleibol, basquetebol e handebol) e grupo sem características de salto ( $n=44$, atletas de futsal e futebol). Para avaliação do desempenho muscular dos flexores e extensores do joelho, foi utilizado um dinamômetro isocinético na velocidade de $60 \% \mathrm{~s} \mathrm{em}$ modo concêntrico. As variáveis analisadas foram pico de torque normalizado pela massa corporal (PT), trabalho total (TT), potência (POT) e relação agonista/antagonista $(I: Q)$. Para o cálculo dos déficits unilaterais, considerouse os valores da relação I:Q inferiores a 50\% e para os déficits bilaterais, assimetrias superiores a 10\% do PT entre os membros. Os resultados indicaram que não houve diferenças significativas no desempenho dos músculos flexores e extensores de joelho entre os grupos, membros e sua interação. Ao comparar déficits significativos de PT e na relação $I: Q$, um maior número de atletas do grupo salto apresentou desequilíbrios na relação $I: Q$ no membro dominante (58,6\% versus $25 \%, p=0,001)$. Na comparação intragrupo, o grupo de atletas sem características de salto apresentou diferença entre os membros nas variáveis PT
\end{abstract}

de extensores e flexores, na POT de flexores e na razão I:Q. Os atletas jovens praticantes de esportes coletivos, independente do grupo, apresentaram assimetrias musculares, e sugere-se a inserção de programas de prevenção de lesão a fim de minimizá-las.

Descritores | Lesões Esportivas; Força Muscular; Joelho; Atletas.

Abstract I This study aims to compare the performance of
knee flexor and extensor muscles in young athletes of team
sports with and without jumping as specific movement. There
were two groups with young male athletes: the jumping
group ( $\mathrm{n}=46$, volleyball, basketball and handball athletes)
and group without jumping characteristics ( $\mathrm{n}=44$, futsal and
soccer athletes). To evaluate the muscle performance of the
flexors and extensors of the knee, we used an isokinetic
dynamometer at a speed of $60^{\circ} / \mathrm{s}$ in concentric mode. The
variables analyzed were peak torque normalized by body
mass (PT), total work (TW), power (POW) and agonist/
antagonist ratio (I:Q). To estimate unilateral deficits, we
considered the values of the I:Q ratio less than $50 \%$, and for
bilateral deficits, asymmetries between the limbs greater
than $10 \%$ for PT. There were no significant differences in the
performance of knee flexor and extensor muscles between
the groups, limbs and their interaction. When comparing

'Universidade Federal do Pampa (Unipampa) - Uruguaiana (RS), Brasil. E-mail: magroliano@hotmail.com. Orcid: 0000-0002-1454-2455 2Universidade Federal do Pampa (Unipampa) - Uruguaiana (RS), Brasil. E-mail: lipt19@yahoo.com.br. Orcid: 0000-0001-7546-1942 ${ }^{3}$ Faculdade Metodista Centenário (FAMES) - Santa Maria (RS), Brasil. E-mail: jjuzinhafisio83@gmail.com. Orcid: 0000-0002-9114-7571 ${ }^{4}$ Universidade Federal de Santa Maria (UFSM) - Santa Maria (RS), Brasil. E-mail: michele.saccol@ufsm.br. Orcid: 0000-0002-7894-690X 5Universidade Federal do Pampa (Unipampa) - Uruguaiana (RS), Brasil. E-mail: simonelara@unipampa.edu.br.

Orcid: 0000-0003-0745-4964 
significant deficits in PT and in the I:Q Ratio, a greater number of athletes in the jumping group showed imbalances in the $\mathrm{I} Q \mathrm{Q}$ ratio in the dominant limb (58.6\% versus $25 \%, p=0.001)$. In the intragroup comparison, the group of athletes without jumping characteristics showed a difference between the limbs in the variables PT of extensors and flexors, in the flexors POW and in the $I: Q$ ratio. Young athletes practicing team sports, regardless of the group, showed muscle asymmetries, and we suggest the insertion prevention programs in order to minimize them.

Keywords | Sport Injuries; Muscle Strength; Knee; Athletes.

Resumen I El objetivo de este estudio fue comparar el desempeño de los músculos flexores y extensores de la rodilla en atletas jóvenes de deportes de equipo que utilizan o no el movimiento de salto como gesto característico. Se incluyeron a jóvenes deportistas masculinos que se dividieron en dos grupos: grupo de salto ( $n=46$, deportistas de voleibol, baloncesto y balonmano) y grupo sin características de salto ( $n=44$, fútbol sala y fútbol). Para evaluar el rendimiento muscular de los flexores y extensores de la rodilla se utilizó un dinamómetro isocinético a una velocidad de $60 \%$ en modo concéntrico. Las variables analizadas fueron torque pico normalizado por masa corporal (PT), trabajo total (TT), potencia (POT) y relación agonista/antagonista (I:Q). Para el cálculo de los déficits unilaterales, se consideraron los valores de la relación I:Q inferiores al 50\%, y para los déficits bilaterales, asimetrías mayores al 10\% del PT entre los miembros. Los resultados indicaron que no hubo diferencias significativas en el desempeño de los músculos flexores y extensores de la rodilla entre los grupos, los miembros y su interacción. Al comparar déficits significativos en PT y en la relación I:Q, un mayor número de atletas en el grupo de salto mostró desequilibrios en la relación I:Q en la extremidad dominante (58,6\% versus $25 \%, p=0,001$ ). En la comparación intragrupo, el grupo de deportistas sin características de salto mostró diferencia entre las extremidades en las variables PT de extensores y flexores, en la POT de flexores y en la relación I:Q. Los deportistas jóvenes que practican deportes de equipo, independientemente del grupo, presentaron asimetrías musculares, por lo que se sugiere la inserción de programas de prevención de lesiones para minimizarlas. Palabras clave I Lesiones Deportivas; Fuerza Muscular; Rodilla; Atletas.

\section{INTRODUÇÃO}

A prática de esportes desenvolve uma melhor aptidão física, melhora o desempenho motor, a socialização e a autoestima, levando cada vez mais os jovens atletas a prática dessas atividades ${ }^{1}$. Apesar desses benefícios, a maior exposição do atleta a constantes gestos repetitivos e sobrecargas, pode levar o mesmo a sofrer algum tipo de lesão musculoesquelética ${ }^{2}$. Nesse sentido, os atletas de esportes de contato estão mais susceptíveis a lesões, decorrentes tanto da repetitividade do movimento quanto das colisões resultantes dos gestos esportivos ${ }^{3}$. Dentre esses movimentos, o salto vertical é um movimento de rápida ação muscular excêntrica seguida por contração concêntrica máxima, demandando grande capacidade de geração de força e trabalho muscular ${ }^{4}$, representando um movimento básico em esportes como handebol, voleibol e basquetebol.

Contudo, estudos envolvendo essas modalidades alertam para o fato da relação existente entre o salto vertical e o desenvolvimento de lesões. No caso do voleibol, a maioria das lesões ocorrem durante o ato de saltar ${ }^{5}$. Entre jogadores de basquete recreacionais, a força de reação do solo durante o salto vertical constitui um fator de risco significativo para o desenvolvimento de lesões musculoesqueléticas ${ }^{6}$.
Corroborando esses achados, alterações biomecânicas na capacidade de salto e de força muscular podem ser encontradas em atletas de handebol com lesão no ligamento cruzado anterior ${ }^{7}$. Vale ressaltar que a quantidade de saltos é variável em cada modalidade esportiva, de forma que o atleta de futebol salta em média apenas 09 saltos por partida, já o jogador de basquete apresenta média de 65 saltos $^{8}$ e o atleta de voleibol salta 150 vezes $^{9}$.

Nesse sentido, a participação em atividades esportivas intensas leva à adaptações específicas, que podem gerar alterações musculoesqueléticas e assim causar mudanças tanto estáticas quanto dinâmicas (estabilidade articular e coordenação neuromuscular, por exemplo), predispondo o atleta à lesões ${ }^{10}$. Com base nisso, se torna importante avaliar a força dos músculos envolvidos no salto, uma vez que essa valência permite identificar indivíduos que se encontram em grupo de risco para o desenvolvimento de lesões musculoesqueléticas ${ }^{11}$.

Os dados obtidos por meio do dinamômetro isocinético são úteis para caracterizar o estado muscular, pois fornecem informações sobre força, potência, resistência e razão agonista/antagonista (I:Q), e têm sido relatados em estudos em diferentes modalidades esportivas ${ }^{12}$. Assim, o objetivo do presente estudo foi comparar o desempenho dos músculos flexores e 
extensores de joelho em atletas de esportes coletivos com salto versus sem características de salto.

\section{METODOLOGIA}

\section{Amostra}

Estudo transversal, descritivo e quantitativo, no qual foi incluída uma amostra por conveniência, formada por atletas amadores das categorias masculinas Sub15 e Sub-17 das modalidades de voleibol, basquetebol, handebol, futsal e futebol de campo, competitivos em nível estadual. Para participarem do estudo, os atletas deveriam ser praticantes de alguma modalidade esportiva supracitada por um período mínimo de 06 meses e estar em treinamento regular, realizando apenas educação física escolar como outra atividade física além de seu esporte competitivo. Os critérios de exclusão foram história de cirurgia ortopédica em membros inferiores e a presença de lesões prévias nos membros inferiores nos últimos 12 meses, relatada pelo atleta ou por meio de laudo médico. Os responsáveis legais assinaram o termo de consentimento livre e esclarecido (TCLE).

Foram coletados dados gerais como a idade, tempo de treinamento, bem como foram avaliados dados antropométricos (massa corporal e estatura).

Os jovens foram alocados em dois grupos, sendo o grupo salto (atletas que praticavam o voleibol, basquetebol e handebol, $n=46$ ) e o grupo sem características de salto, constituído pelos atletas que não tinham o salto como característico do esporte (futsal e futebol de campo, $n=44$ ).

\section{Instrumentos}

A performance muscular foi avaliada por meio de um dinamômetro isocinético (Biodex-System PRO 4). Antes de iniciar o teste, os sujeitos realizaram um aquecimento de cinco minutos em uma bicicleta ergométrica, sem carga. Em seguida, foram posicionados no dinamômetro, estabilizados com cintos no tronco e na coxa para evitar movimentos compensatórios, e executaram três repetições submáximas prévias para familiarização com os procedimentos ${ }^{13}$. Após, realizaram cinco repetições máximas de flexão e extensão do joelho bilateralmente, com intervalo de um minuto entre as séries, no qual a escolha do primeiro membro a ser testado foi feita de forma aleatória. Foi selecionado o modo concêntrico-concêntrico na velocidade de $60^{\circ} \%$ e os atletas receberam estímulo verbal para desenvolver força máxima a cada repetição ${ }^{13}$. Essa velocidade angular foi selecionada, pois ela é a indicada para o recrutamento de um maior número de unidades motoras, possibilitando uma melhor representação do trabalho máximo realizado pela musculatura avaliada ${ }^{13}$.

As variáveis pico de torque normalizado pela massa corporal (PT), trabalho total (TT) e potência (POT) foram analisadas, bem como a relação agonista/ antagonista (I:Q $)^{14}$. Para o cálculo dos déficits, os valores de normalidade da relação I:Q foram considerados de $60 \%{ }^{15}$, sendo que valores abaixo de $50 \%$ indicam grau severo de desequilíbrio muscular ${ }^{16}$. Em relação aos valores ideais para comparações bilaterais, assimetrias superiores a 10\% entre os membros são consideradas risco elevado para lesões ${ }^{15}$ e foram consideradas como déficits.

\section{ANÁLISE ESTATÍSTICA}

Para a análise estatística, utilizou-se o programa SPSS, versão 20.0, com análise descritiva, por meio de medidas de média e desvio-padrão. Para testar a normalidade dos dados, foi realizado o teste Shapiro-Wilk. Os dados seguiram uma distribuição normal e a comparação entre grupos (salto e sem características de salto) e membros (dominante e não dominante) foi realizada pela Anova de dois fatores (grupo e membro). Foi realizado teste t para comparação intragrupos. Além disso, para comparação das frequências de déficits do membro dominante nos extensores e flexores, bem como o percentual de desequilíbrios de I:Q nos grupos, o teste Qui-quadrado foi utilizado. Para todas as análises foi considerado um nível de significância de 0,05.

\section{RESULTADOS}

A caracterização da amostra está presente na Tabela 1.

Tabela 1. Caracterização da amostra. Dados apresentados em média e desvio-padrão ( \pm DP)

\begin{tabular}{lccr} 
Variável & $\begin{array}{c}\text { Grupo salto } \\
\mathrm{n}=46\end{array}$ & $\begin{array}{c}\text { Grupo sem } \\
\text { características de salto } \\
\mathbf{n}=44\end{array}$ & $\mathbf{p}$ \\
\hline Idade (anos) & $15,02 \pm 1,10$ & $14,98 \pm 1,17$ & 0,68 \\
Massa (kg) & $67,64 \pm 13,27$ & $63,12 \pm 10,47$ & 0,12 \\
Estatura (m) & $1,73 \pm 0,08$ & $1,67 \pm 0,06$ & $<0,01$
\end{tabular}

A Tabela 2 evidencia o desempenho dos músculos flexores e extensores de joelho no membro dominante 
e não dominante dos atletas avaliados. A comparação entre grupos, membros e sua interação não revelou diferenças significativas.

Ao comparar déficits musculares entre os grupos, uma maior frequência de atletas do grupo salto $(58,6 \%)$ apresentou desequilíbrios na relação I:Q no membro dominante, quando comparado ao grupo sem características de salto (25\%), e tal diferença entre os grupos foi significativa $(p=0,001)$.
$\mathrm{Na}$ comparação intragrupo, o grupo de atletas sem características de salto apresentou diferença entre os membros nas variáveis $\mathrm{PT}$ de extensores e flexores, na POT de flexores e na razão I:Q. O PT dos extensores foi superior no membro dominante ( $\mathrm{p}=0,031)$, enquanto que as variáveis de PT ( $\mathrm{p}=0,011)$ e POT $(\mathrm{p}=0,01)$ dos flexores foram superiores no membro não-dominante. A relação I:Q também foi superior no membro não dominante dos atletas que não realizam saltos.

Tabela 2. Pico de torque (PT em N.m/kg), trabalho total (TT em J), potência (POT em W), relação de equilíbrio muscular (I:Q em \%) e déficits (em \%) de flexores e extensores de joelho em atletas que realizam salto versus sem características de salto. Dados apresentados em média e desvio-padrão ( \pm DP) e percentual

\begin{tabular}{|c|c|c|c|c|c|c|c|}
\hline & \multicolumn{2}{|c|}{$\begin{array}{l}\text { Grupo salto } \\
n=46\end{array}$} & \multicolumn{2}{|c|}{$\begin{array}{l}\text { Grupo sem características de salto } \\
\qquad n=44\end{array}$} & \multicolumn{3}{|c|}{ Anova } \\
\hline & Dominante & Não dominante & Dominante & Não dominante & Grupo & Membro & Interação \\
\hline PT extensores & $253,86 \pm 53,91$ & $248,1 \pm 54,11$ & $271 \pm 54,82^{*}$ & $260,78 \pm 45,62$ & 0,933 & 0,456 & 0,903 \\
\hline Déficit PT extensor ${ }^{a}$ & \multicolumn{2}{|c|}{45,65} & \multicolumn{2}{|c|}{43,18} & & & \\
\hline PT flexores & $134,38 \pm 36,37$ & $130,99 \pm 55,42$ & $135,26 \pm 29,02$ & $141,80 \pm 29,11^{*}$ & 0,177 & 0,794 & 0,462 \\
\hline Déficit PT flexora & \multicolumn{2}{|c|}{30,43} & \multicolumn{2}{|c|}{38,63} & & & \\
\hline TT extensores & $753,30 \pm 208,83$ & $741,28 \pm 201,11$ & $713,30 \pm 208,67$ & $683,02 \pm 187,67$ & 0,104 & 0,483 & 0,762 \\
\hline TT flexores & $402,72 \pm 111,48$ & $390,11 \pm 105,48$ & $384,37 \pm 133,43$ & $388,03 \pm 124,28$ & 0,332 & 0,801 & 0,647 \\
\hline POT extensores & $109,28 \pm 29,08$ & $108,6 \pm 33,35$ & $111,35 \pm 32,79$ & $107,49 \pm 28,94$ & 0,972 & 0,726 & 0,631 \\
\hline Déficit POT extensores ${ }^{a}$ & \multicolumn{2}{|c|}{50} & \multicolumn{2}{|c|}{52,27} & & & \\
\hline POT flexores & $58,28 \pm 16,26$ & $57,1 \pm 15,88$ & $58,63 \pm 18,57$ & $61,88 \pm 19,07^{*}$ & 0,250 & 0,532 & 0,554 \\
\hline Déficit POT flexoresa & \multicolumn{2}{|c|}{47,82} & \multicolumn{2}{|c|}{43,18} & & & \\
\hline Razão I:Q & $52,13 \pm 8,67$ & $52,16 \pm 13,98$ & $50,50 \pm 7,82$ & $54,55 \pm 6,97^{*}$ & 0,791 & 0,164 & 0,170 \\
\hline Desequilíbrio I:Qb & $58,69^{+}$ & 45,65 & 25 & 52,27 & & & \\
\hline
\end{tabular}

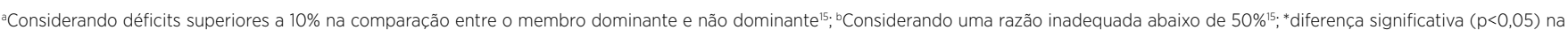
comparação intragrupos; ${ }^{+}$diferença significativa na comparação intergrupos $(p=0,001)$

\section{DISCUSSÃO}

Não houve diferença em relação ao desempenho dos músculos flexores e extensores de joelho em jovens atletas de esportes coletivos de salto versus sem salto. Porém, um percentual maior de atletas do grupo salto apresentou razão I:Q do membro dominante abaixo dos valores de normalidade, o que pode aumentar sua chance de lesão. Apesar dessa diferença, é interessante observar que um grande número de atletas em ambos os grupos apresentou valores inferiores aos de referência, que é de aproximadamente $60 \%{ }^{15}$ nas velocidades angulares baixas. Razões I:Q abaixo de 50\% são consideradas desequilíbrios musculares importantes ${ }^{16}$, tornando-se um alto fator de risco para lesões esportivas. Estudos prévios já identificaram que atletas com razões inferiores a $50,5 \%$ aumentam a chance de lesões musculares agudas ${ }^{17}$, especialmente os jogadores de futebol ${ }^{18}$.

No estudo de Magalhães et al. ${ }^{19}$, atletas de voleibol apresentaram menor razão I:Q no membro não dominante quando comparados aos jogadores de futebol, avaliados na velocidade de $90 \%$, diferentemente do nosso estudo, em que não houve diferença entre os grupos de atletas. Contudo, cabe ressaltar que os atletas avaliados por Magalhães et al. ${ }^{19}$ eram da categoria adulta e profissional, além da diferença na velocidade utilizada para a avaliação isocinética. Sugere-se que esses fatores possam explicar as diferenças encontradas entre os estudos. Por outro lado, Andrade et al. ${ }^{20}$, ao comparar atletas de futebol, judô e handebol masculino, identificou que os judocas apresentaram uma razão I:Q inferior aos jogadores de futebol, sem diferenças entre os jogadores de handebol e futebol, o que vai ao encontro dos dados de nosso estudo.

Nesse estudo, podemos verificar que o grupo de atletas sem características de salto apresentou assimetrias significativas entre membros em relação ao PT de extensores e flexores, POT de flexores e razão I:Q. Essas assimetrias no grupo dos atletas de futebol e futsal podem estar associadas às características fundamentais dessas modalidades, que apresentam padrões cinéticos assimétricos ${ }^{21}$. Fousekis, Tsepis e Vagenas ${ }^{21}$ encontraram importantes assimetrias quanto à performance muscular 
nos membros inferiores de jogadores jovens de futebol, possivelmente por ocorrer adaptações assimétricas durante o jogo. Ainda, os autores chamam a atenção para que medidas preventivas sejam inseridas nessa modalidade, a fim de corrigir as assimetrias de força, através da modificação individual da carga de treinamento.

Além da avaliação isocinética prover dados referentes a fatores de risco para possíveis lesões musculoesqueléticas, essa avaliação também é relevante no contexto da performance esportiva. Nesse aspecto, ambos os grupos apresentaram assimetrias superiores a $10 \%$ entre os membros na POT de extensores e flexores de joelho. Conforme Fonseca et al. $^{22}$, déficits em potência na velocidade baixa podem influenciar o desempenho no movimento de arrancada durante uma partida, e, portanto, esse fator deve ser corrigido nos treinamentos, a fim de evitar que o atleta perca desempenho. Nesse caso, é importante que o trabalho de desenvolvimento de POT especialmente de extensores do joelho seja realizado, a fim de evitar perda de rendimento esportivo.

Com base nesses dados, sugere-se a relevância da inserção de programas de prevenção de lesão em atletas jovens, que visam reduzir as assimetrias uni e bilaterais. A inserção desses programas voltados para atletas jovens é de extrema importância, pois, conforme Atkins et al. ${ }^{23}$, os desequilíbrios musculares, especialmente bilaterais, se tornam marcantes no início da adolescência, e ao final desse estágio, essas diferenças parecem reduzir. Dessa forma, sugerem que um treinamento específico, voltado para amenizar os desequilíbrios musculares de membros inferiores em jogadores jovens contribui para a redução de risco de lesões nessas categorias.

Como contribuição, os dados sobre performance muscular apresentados nesse estudo podem ser utilizados como valores de referência na prevenção, treinamento e reabilitação de jovens atletas.

\section{CONCLUSÃO}

Não houve diferença no desempenho dos músculos flexores e extensores de joelho em atletas de esportes coletivos com e sem características de salto. Porém, o grupo salto apresentou maior número de atletas com desequilíbrios musculares severos na razão $\mathrm{I}: \mathrm{Q}$ no membro dominante.

Nas comparações intragrupos, os atletas de futebol e futsal apresentaram maior PT no grupo extensor dominante e nas variáveis de flexores do membro não dominante.
Com base nesses achados, essas modalidades esportivas parecem influenciar os valores de performance muscular.

\section{REFERÊNCIAS}

1. Wenger DRMD. The Sports Medicine Bible for Young Athletes. J Pediatr Orthop. 2002;22(4):559. doi: 10.1097/01241398-200207000-00033

2. Knowles SB, Marshall SW, Bowling JM, Loomis D, Millikan $\mathrm{R}$, Yang J, et al. A prospective study of injury incidence among North Carolina High School Athletes. Am J Epidemiol. 2006;164(12):1209-21. doi: 10.1093/aje/kwj337

3. Santos SG, Piucco T, Reis DC. Fatores que interferem nas lesões de atletas amadores de voleibol. Rev Bras Cineantropom. Desempenho Hum. 2007;9(2):189-95.

4. Lian O, Engebretsen L, Ovrebo RV, Bahr R. Characteristics of the leg extensors in male volleyball players with jumper's knee. Am J Sports Med. 1996;24(3):380-5. doi: 10.1177/036354659602400322

5. Eerkes K. Volleyball injuries. Curr Sports Med Rep. 2012;11(5):2516. doi: 10.1249/JSR.0b013e3182699037

6. Kilic Ö, Van Os V, Kemler E, Barendrecht M, Gouttebarge V. The 'Sequence of Prevention' for musculoskeletal injuries among recreational basketballers: a systematic review of the scientific literature. Phys Sports Med. 2018;46(2):197-212. doi: 10.1080/00913847.2018.1424496

7. Setuain I, Millor N, Alfaro J, Gorostiaga E, Izquierdo M. Jumping performance differences among elite professional handball players with or without previous ACL reconstruction. J Sports Med Phys Fitness. 2015;55(10):1184-92.

8. Rocha CM, Ugrinowitsh C, Barbanti VJ. A especificidade do treinamento e a habilidade de saltar verticalmente: um estudo com jogadores de basquetebol e voleibol de diferentes categorias. Lect EF Deportes. 2005;10(84):[1 p.].

9. Rocha MA. Quantificação do número de saltos de ataque, bloqueio e levantamento no voleibol feminino [dissertação]. Universidade de São Paulo: São Paulo; 2010.

10. Siqueira CM, Pelegrini FR, Fontana MF, Greve JM. Isokinetic dynamometry of knee flexors and extensors: comparative study among non-athletes, jumper athletes and runner athletes. Rev Hosp Clin Fac Med Sao Paulo. 2002;57(1):19-24.

11. Silva Neto M, Simões R, Grangeiro Neto JA, Cardone CP. Avaliação isocinética da força muscular em atletas profissionais de futebol feminino. Rev Bras Med Esporte. 2010;16(1)33-5. doi 10.1590/S1517-86922010000100006

12. Andrade MS, Vancini RL, de Lira CA, Mascarin NC, Fachina RJ, da Silva AC. Shoulder isokinetic profile of male handball players of the Brazilian National Team. Braz J Phys Ther. 2013;17(6):572-8. doi: 10.1590/S1413-35552012005000125

13. Bittencourt NFN, Amaral GM, Anjos MTS, D’Alessandro R, Silva AA, Fonseca ST. Avaliação muscular isocinética da articulação do joelho em atletas das seleções brasileiras infanto e juvenil de voleibol masculino. Rev Bras Med Esporte. 2005;11(6):331-6. doi:10.1590/S151786922005000600005 
14. Terreri AP, Greve JMD, Amatuzzi MM. Avaliação isocinética no joelho do atleta. Rev Bras Med Esporte. 2001;7(5):170-4.

15. Zabka FF; Valente HG; Pacheco AM. Avaliação isocinética dos músculos extensores e flexores de joelho em jogadores de futebol profissional. Rev Bras Med Esporte. 2011;17(3):189-92. doi: 10.1590/S1517-86922011000300008

16. Andrews JR, Harrelson GL, Wilk KE. Reabilitação física das lesões desportivas. 3a ed. Rio de Janeiro: Guanabara Koogan; 2005.

17. Lee JWY, Mok KM, Chan HCK, Yung PSH, Chan KM. Eccentric hamstring strength deficit and poor hamstringto-quadriceps ratio are risk factors for hamstring strain injury in football: A prospective study of 146 professional players. J Sci Med Sport. 2018;21(8):789-93. doi: 10.1016/j. jsams.2017.11.017

18. Ardern CL, Pizzari T, Wollin MR, Webster KE. Hamstrings strength imbalance in professional football (soccer) players in Australia. J Strength Cond Res. 2015;29(4):997-1002. doi: 10.1519/JSC.0000000000000747
19. Magalhães J, Oliveira J, Ascensão A, Soares J. Concentric quadriceps and hamstrings isokinetic strength in volleyball and soccer players. J Sports Med Phys Fitness. 2004;44(2):119-25.

20. Andrade MS, De Lira CA, Koffes FC, Mascarin NC, BeneditoSilva AA, Da Silva AC. Isokinetic hamstrings-to-quadriceps peak torque ratio: the influence of sport modality, gender, and angular velocity. J Sports Sci. 2012;30(6):547-53. doi: 10.1080/02640414.2011.644249

21. Fousekis K, Tsepis E, Vagenas G. Multivariate isokinetic strength asymmetries of the knee and ankle in professional soccer players. J Sports Med Phys Fitness. 2010;50(4):465-74.

22. Fonseca ST, Ocarino JM, Silva PLP, Bricio RS, Costa CA, Wanne LL. Caracterização da performance muscular em atletas profissionais de futebol. Rev Bras Med Esporte. 2007;13(3):143-7. doi: 10.1590/ S1517-86922007000300003

23. Atkins SJ, Bentley I, Hurst HT, Sinclair JK, Hesketh C. The Presence of Bilateral Imbalance of the Lower Limbs in Elite Youth Soccer Players of Different Ages. J Strength Cond Res. 2016;30(4):1007-13. doi: 10.1519/JSC.0b013e3182987044 